\title{
Truth in an Evolutionary Perspective
}

\author{
CARLOS BLANCO \\ Mind-Brain Project. Institute for Culture and Society (ICS). \\ University of Navarra. 31009 Pamplona (Spain) \\ carlosblanco@unav.es
}

"Die Natur... Ichsprachnicht von ihr. Nein, was wahrist, und was falschist, alles hat siegesprochen. Alles ist ihre Schuld, alles ist ihr Verdienst"

(Johann Wolfgang Goethe, “Die Natur”, 1783)

\begin{abstract}
The perspective drawn from evolutionary science, undoubtedly one of the most remarkable intellectual achievements in our conception of the world, poses a deep challenge to epistemology and the meaning of truth. The present paper aims to examine the difficulties offered by the prevailing biological model for the emergence and development of mind in its attempt at constructing a possible philosophical theory of truth. We propose a solution which, while preserving the priority of the distinction between truth and falsehood, is nonetheless capable of reconciling its philosophical fundamentals with the scientific evidence concerning the evolutionary origin of our capacity to recognize truth and falsehood.
\end{abstract}

Keywords: truth; evolution; efficiency; intelligibility. 


\section{The problem}

When we try to apply the important and ineludible evolutionary perspective to the emergence of the most complex psychic faculties of the human species, a deep conceptual challenge arises in a naturalized epistemology ${ }^{1}$. As some authors have highlighted (Street 2006; Copp 2008; Kahane 2011), there is a true "Darwinian dilemma" for any realist theory of value, if by "realist" we understand the philosophical position which defends the independence of value statements from our evaluative attitudes ${ }^{2}$.

According to the theory of evolution, forces such as natural selection have played a key role in shaping our psychic faculties and therefore our capacity to evaluate reality (through the dichotomy "true/false" in the epistemological realm and "good/bad" in the ethical sphere). No unjustified extrapolation would appear if we admitted that evolutionary forces must have influenced our evaluative attitudes, necessarily determined by the natural dynamics that have contributed to their constitution. However, the problem refers not so much to the evolutionary dependence of our evaluative attitudes but to the possible evolutionary independence of value (like truth) as such. Clarifying which type of relationship has existed between naturally evolved evaluative attitudes and the realm of values (in this paper we will focus on the distinction between truth and falsehood) is still a problem to be solved in a convincing way, because the connection between evolutionary forces and our evaluative faculties is not trivial: we do not know if it should be formulated in terms of sufficient or merely necessary conditions.

If naturally evolved evaluative attitudes are the sufficient condition for the existence of value, we must say that evaluative judgments depend on psychic faculties which are inexorably subordinated to the influx of

1 For an overview of evolutionary epistemology and an access to the most relevant literature on the subject, see the homonymous article written by M. Bradie and W. Harms in the Stanford Encyclopedia of Philosophy (Bradie and Harms 2011).

2 Examples of this position can be found in (Nagel 1989; 2012; Dworkin 1996; Shafer-Landau 2003; Enoch 2011). For a comprehensive defense of the possibility of naturalizing epistemology, see (Allen and Leiter 2001). 
evolutionary forces. Therefore, they lack autonomy and values as such do not properly exist. They simply represent exemplifications of the general mechanism governing biological nature: the development of a series of abilities as instruments to satisfy the adaptive pressures exerted by the environment. If naturally, evolved evaluative attitudes are the necessary but not sufficient condition for the existence of value, it is legitimate to defend the following position: although evolutionary forces have served as the basis for the development of certain psychic potentialities, once the latter have emerged, they display a series of properties which cannot be reduced to purely evolutionary causes. Rather, they enjoy a relevant degree of autonomy.

As we can easily notice, an apparently sectorial question opens the window for exploring a deep epistemological matter: the evidence for considering reality as being structured into different levels, some of which could perhaps be irreducible to others. But why do different levels exist? A courageous naturalistic vision must embrace reductionism, even if it recognizes the huge complexity of the "frontiers" that separate the different levels. However, a basic insurmountable irreducibility between certain levels exists (perhaps only in the epistemological sphere), as we will show.

Evolutionary explanations of the realm of human values normally attempt to reduce ideas such as truth and falsehood to their biological foundations. The principal concept, the explanatory master key for achieving this ambitious enterprise, resides in natural selection, a force acting on individual variations in order to favor those which turn to be more advantageous for survival (of the individual?; of the group?; of the species?; of all of them at once?; we do not know) ${ }^{3}$.

For example, some authors have proposed mechanisms regarding the evolution of the human capacity to form metarepresentations. As Sperber writes, "there is a plausible scenario where a metarepresentational ability develops in the ancestral species for reasons having to do with competition,

See (Ball 2013). 
exploitation and co-operation, and not with communication per se. This metarepresentational ability makes a form of inferential communication possible, initially as a side effect, and, probably, rather painstakingly at first. The beneficial character of this side effect turns it into a function of metarepresentations, and creates a favorable environment for the evolution of a new adaptation, a linguistic ability. Once this linguistic ability develops, a co-evolutionary mutual enhancement of both abilities is easy enough to imagine" (Sperber 2000, 129).

According to Darwin's theory of descent with modification (Darwin 1859), advantageous variations will be inherited by the next generations if they facilitate adaptation to the environment: if a trait turns to be heritable, then it will persist in the offspring if it shows a certain degree of utility for adaption. The epistemological presuppositions of this explanatory frame seem clear: the more complex levels are susceptible to reduction into their chronological or physiological "antecedents". If we leave aside the difficulties of this project, in light of its failure in other branches of knowledge $^{4}$, aggravated by the absence of a scientific theory which convincingly explains why reality is structured into levels that maybe irreducible (and why new properties emerge in the whole that do not exist in its parts ${ }^{5}$ ), we will indicate how unfruitful it is to try to reduce the realm of values into evolutionary forces. Its failure reveals, in our view, a deep epistemological gap within the universe of human knowledge. But before continuing, we want to make clear that we do not embrace any kind of "mystical" approach to nature which fragments reality into irreducible levels. We believe that all levels of complexity ultimately result from the power of matter to evolve over time and achieve higher degrees of development in the physical, chemical, biological, and psychological realms. Rather, we aim to show that there is an epistemological need for distinguishing between different

4 Mathematics cannot be reduced into Logic (Gödel 1931), and Biology is hardly reducible into Physics and Chemistry, since concepts such as natural selection and the famous distinction between Umwelt and Innenwelt in living beings are difficult to express in purely physical-chemical terms.

5 The examination of how the binomial integrated by "parts" plus "interaction" transforms parts themselves may constitute the explanatory key to this difficulty. 
levels, but this requirement does not probably involve any kind of ontological prohibition.

\section{Truth or efficiency?}

Let us admit that truth could be interpreted as a mere adaptive circumstance, id est, as the way in which a certain species navigates its own world. Then, it would be legitimate to state:

For species $\mathrm{S}(\mathrm{A})$, truth (its "world view") is equivalent to T(A).

For species $S(B)$, truth (its "world view") is equivalent to $T(B)$.

But how could we commensurate $\mathrm{T}(\mathrm{A})$ and $\mathrm{T}(\mathrm{B})$ if both $\mathrm{S}(\mathrm{A})$ and $\mathrm{S}(\mathrm{B})$ experienced the same world? According to an orthodox evolutionary paradigm of truth, $\mathrm{T}(\mathrm{A})$ and $\mathrm{T}(\mathrm{B})$ should respond to different world views. However, if the perception of truth were the byproduct of evolutionary forces (not an independent frame which we can grasp through evolutionary forces), in a given time and confronted with the same phenomenon, two truths would coexist: $\mathrm{T}(\mathrm{A})$ and $\mathrm{T}(\mathrm{B})$. If we leave aside the degree of complexity possessed by each species A and B, both truths should be identically valid. But even if they referred to phenomena equally "perceptible" for both species, there should be a criterion capable of establishing which truth is correct. Let us suppose that a chimpanzee believes that there is a predator in a position in which someone has placed the hologram of a lion. Besides the chimpanzee, a man who knows that there is no lion at all, but only a holographic representation (which, if we take as criterion of truth actual physical existence, cannot be considered a true lion), stands. Let us suppose that for the chimpanzee $\mathrm{T}(\mathrm{A})$ differs from $\mathrm{T}(\mathrm{B})$. $\mathrm{T}(\mathrm{A})$ and $\mathrm{T}(\mathrm{B})$ cannot not be true at the same time if they contain opposite claims, for the principle of non-contradiction would be violated and anything could hold.

If truth were identified with efficiency, so that scientific explanations were true inasmuch as they were efficient and helped us adapt ourselves to our own world in a satisfactory manner, Einstein's theory of relativity would not be provisional in the same way as it is for Popper (as the result of the impossibility that human knowledge faces in evaluating all possible 
situations and all possible outcomes), but because it constitutes a particular adaptation, fulfilling the same evolutionary role than the "worldview" endorsed by any animal species. However, several problems emerge from this assumption. Superstition might be regarded as an efficient adaptation to a world which had not achieved a specific degree of social and economic development, never as an intrinsically false perspective concerning science and empirical truth. Of course, any relativistic, anti-scientific approach to the study of the world should be accepted, and from this "epistemological anarchism" of Feyerabendian resonances (Feyerabend 1975) no serious grounding of the scientific method, perhaps the pinnacle of human intellect, could be offered.

Let us examine the several difficulties arising from the identification of truth with biological efficiency. First of all, how is it possible that a particular adaptation, which could be surpassed by a more efficient one in the future, may discover the fundamental laws of the universe? The question concerning the truth of a scientific theory would be meaningless: it would be true as long as it were "useful" for explaining certain empirical observations. Leaving aside logical and mathematical truths ("self-sufficient" adaptations whose realm of action would be constrained to the human mind; they would not enjoy universality), it is extremely implausible to think that a scientific theory (for example, the theory of chemical elements) constitutes a mere efficient adaptation to the environment. Those chemical elements really exist, not only on the basis of scientific methodology (precisely those canons which validate or refute Darwin's theory and any evolutionary approach), but of reality itself. Popper (1935) establishes a necessary theoretical limit for scientific statements, but our observation that the Earth rotates around the Sun is indisputable (general relativity explains that it is the Earth that "falls" towards the Sun due to the latter curving the space more intensely). Of course, any scientific theory can be refined and improved towards some sort of "asymptotic limit." Newtonian mechanics, which seemed the culmination of the scientific endeavor of mankind, succumbed to quantum mechanics and relativity in the early $20^{\text {th }}$ century. However, and in spite of this apparently endless "internal 
progress" within each theory, we can say that we have discovered a truth about nature, as incomplete as it may seem ${ }^{6}$. If it were true only in accordance with scientific canons, why should we accept it? Just because of its functionality? We would lack any serious epistemological reason to prefer Newtonian over Aristotelian physics. As long as a more refined model has not been proposed, we have no reason to deny the reality (beyond its circumscription to a certain theoretical model) of entities such as electrons and quarks.

It seems clear that if we renounce a criterion of truth, a full range of epistemological incongruities emerges, for a constructivist view of truth faces serious difficulties (not so much in the ontological as in the epistemological sphere). First of all, it does not explain why new levels flourish. Secondly, it presupposes the possibility of reducing higher into lower levels, although this goal has not been factually achieved: deep fissures exist within nature and mind, as shown by Gödel's theorem ${ }^{7}$ and the irreducibility of the basic levels of reality into a fundamental "determination" (according to Heisenberg's principle: the existence of processes which are not computable is highlighted by quantum mechanics ${ }^{8}$ ).

On the contrary, since it is clear, in the light of biological evidence, that our capacity for grasping truth is the result of evolutionary processes, so that our perception of reality depends upon the degree of development achieved by our nervous system (mediated, to a huge extent, by forces such as natural selection), an acceptable convincing link between truth and evolutionary processes must be proposed. But if this hypothetical connection involved negating of the autonomy of truth, now subsumed into efficiency, insurmountable difficulties would originate and no fruitful epistemological result could be conquered.

6 Finite progress does not dissolve into infinite progress. A finite truth is a real truth. Against Hegel, it is not necessary to know the whole truth to be sure that we have reached a real, finite truth. Cantor's work on the theory of transfinite numbers has clearly shown that it is possible to conceive of infinite yet numerable series (Cantor 1915).

7 On the difficulties that Gödel's theorem poses to artificial intelligence and the theory of mind, see (Lucas 1961).

8 On the importance of quantum indeterminacy for epistemology, see (Sols 2014). 
In addition to the former epistemological problems, we should also notice that the consecration of biological efficiency as explanatory criterion poses great challenges. First of all, we do not know exactly over which units it is applied. Secondly, sometimes that which is successful involves the elimination of part of the descent: the success of some individuals, groups, or species generally implies the failure of others. The "counter-argument" holding that this scenario confirms natural selection falls into circularity: the statement that only those individuals which become adapted in the most efficient way survive does not clarify much about how to elucidate $e x$ ante the most efficient adaptation, whereas unfolding it ex post succumbs to circularity. Thirdly, why is altruism possible? Why does not every individual look for its own goals in terms of biological success? A posteriori, everything is successful, if success is measured in terms of rates of survival. But this realization is merely descriptive if not tautological. Were biological efficiency prior to truth in the epistemological realm, the world could be fragmented into as many "truths" as species, groups, or even individuals we could characterize.

No one would apply natural selection as the explanatory principle for justifying logical and mathematical truths. In the same way as no one would examine history, politics, and art only from the criterion of biological efficiency, as important as it may be for that which Ferdinand Braudel called "la longue durée" (Braudel 1958), it is hard to understand why it should play a predominant role in the birth of consciousness. If we accept the emergence of levels, we must commit ourselves to the idea that laws absent in lower levels can (at least potentially) arise in more complex levels. Biological systems do not violate the principle of conservation of energy, but on the sole basis of physical laws we will not be able to explain life and its adaptations. We need new principles, which do not rule over purely physical systems: natural selection, teleonomy... For example, Von Uexküll's distinction between Innenwelt and Umwelt is not relevant (perhaps only functionally, but not fundamentally) in non-biological systems. The very idea of "selection" introduces a key conceptual novelty in biology. 
There is no reason to suppose that natural selection preserves its explanatory power in the realm of human consciousness, in particular in its perception of truth. If the existence of different levels within reality seems to exhort us to respect their own frontiers and the epistemological principles ruling each of them, the abuse of natural selection ${ }^{9}$ in realms other than the biological development of organisms should be considered an unwarranted extrapolation. However, and since refined versions of Darwin's theory constitute the only scientifically plausible model to explain the development of life, we are left with two propositions in order to reconcile the primacy of biological efficiency in evolutionary forces with the epistemological priority of truth:

1) Darwin's theoretical outcomes are incomplete but they still offer a global framework, which should be progressively refined.

2) The intelligibility of the world is consubstantial to the world itself and it only becomes patent once the central nervous system has achieved a certain degree of complexity.

The idea that our evaluative capacities have emerged "because they forged adaptive links between our ancestors' circumstances and their responses to those circumstances" (Street 2006,127) offers little explanatory power: why these links instead of others? Why should biological efficiency allow the appearance of falseness? What empirical proofs do we have to support this claim as a universal truth? How can we demonstrate that a certain evaluative attitude has been selected, if we can imagine different and even more successful evaluative attitudes that might have been generated? We inherit the capacity to evaluate, not specific evaluations, which must be learned or created by every individual. Because specific evaluative attitudes are learnt instead or created of being inherited, they cannot be selected by nature. The notion that nature selects flexible evaluative attitudes is too vague and it is not able to explain why we evaluate A instead of B in a particular situation: it may justify why we have "evaluative capacities",

9 For a classical critique of the adaptationist programme in biology ("based on faith in the power of natural selection as an optimizing agent”), see (Gould and Lewontin 1979), in which a pluralistic account of functionality and evolutionary account is proposed. 
but it does not vindicate why we adopt this or that evaluative attitude (its normativity). Also, we should notice that evaluative judgments are never automatic, at least if we achieve them through reflection (and we can always, at least as a theoretical limit, reach them through reflection), for we can always distinguish A from B. Therefore, they cannot be interpreted as mere "reflexes", but as conscious acts.

\section{A plausible solution}

In our opinion, the only convincing way of naturalizing truth demands that we attribute both ontological and epistemological priority to this notion (truth is equivalent to the world qua intelligible, but its intelligibility stems from its own "logical" structure), gradually perceived, with deeper degrees of insight, as the development of the nervous system propitiates the appearance of intelligence (the faculty of reasoning in order to achieve truth).

If, in imperiously simplified terms, the principle guiding the evolution of biological species, the motor force behind the expansion of the tree of life, can be summarized as "adaptive efficiency", which should be understood in terms of reproductive success, it is enormously difficult to justify the possibility of human judgments about truth and falseness from this basis alone. Truth would be constrained to an adaptive context, and it would never become independent from this "temporally efficient" biological niche under whose constraints it has emerged. However, this position leads us into absurdities. If we accept it, we do not possess any valid conceptual tool to elucidate whether the theory of evolution is true or false without succumbing to circularity. We are forced to consider it a "self-contained" explanatory frame. Therefore, it becomes unfalsifiable ${ }^{10}$. The existence of science demands careful respect for the independence of the duality "truth/ falsehood" in any explanation (not a mere description) which deserves to be called "scientific."

10 For a discussion on the unfalsifiability of evolutionary psychology, see (Stamos 1996; Ketelaar and Ellis 2000). 
Our knowledge of natural history and genetics has offered undisputable evidence for accepting that species have evolved over time through purely natural causes. Both the scientist and the philosopher face an ineludible dilemma: evolutionary forces have shaped the development of species and they have ultimately led to mankind ${ }^{11}$. We cannot analyze the explanatory leaks that still persist in current models of evolutionary theory, such as the probable insufficiency of the binomial "random genetic mutations" (source of variation) and natural selection for explaining the origin of new species, the emergence of biological novelty (paradigms such as "facilitated variation", Kirschner and Gerhart 2009, seem to refine the Neodarwinian synthesis through the incorporation of the latest discoveries on epigenetics and developmental biology). Also, as we have already mentioned, we do not fully understand how natural selection works: does it act upon genes, individuals, groups..., or upon all of them at once, although this process could generate formidable incoherencies? Rather, we claim the necessity of establishing a connection between evolutionary forces (the biological basis for the development of human psychic faculties) and the independence exhibited, prima facie, by the objects of certain psychic faculties.

If evolutionary forces can be condensed into the principle of "primacy of biological efficiency" and the world of human knowledge is based upon the distinction between truth and falsehood, it is neither possible to reduce the latter into the former nor to exclude the former from the explanation of the latter. If the distinction between truth and falsehood depends upon evolutionary forces, we cannot elucidate whether the scientific theory created to explain evolutionary forces is true or false. Strange authorities would compel us to accept the theory of evolution as a totalizing, not contingent and self-contained explanation. However, this position violates basic criteria of scientific legitimacy. Anything would hold, for any

11 We cannot know if this is a necessary outcome: we simply observe that humanity has emerged and we learn from our observation of the world that it constitutes the pinnacle of evolutionary complexity; whether this process is purely random or ineluctable cannot be decided on scientific grounds (Sols 2014), although evidence for some kind of "inexorable path" (some kind of "orthogenesis") within evolutionary dynamics is in no ways conclusive. 
alternative theory claiming to be prior to the distinction between truth and falsehood could also be admitted on no other grounds that its own power of persuasion. But, on the contrary, if we exclude evolutionary forces from the explanation of the highest psychic faculties of the human being, we deny a vast array of empirical evidence. How can we escape from this labyrinth?

We think that the only convincing solution lies in defending that evolutionary forces, guided by the primacy of biological efficiency, have led us into the most efficient adaptation: that which allows certain individuals to grasp the deepest nature of the surrounding world with increasing degrees of insight. In any case, the perception of the "reality" of the world must enjoy independence from the criterion of purely biological efficiency; otherwise, efficiency could not be actually achieved, for, eclipsed by the search of the highest adaptive success, no "independent" picture of the surrounding reality would be captured by those individuals. As a consequence, obtaining the most efficient adaption to the reality to which they must become adapted would not be guaranteed. An adaptation capable of providing us with a more adjusted, deep, and "amplifiable" knowledge of the surrounding environment will always be more efficient. Grasping "truth" is extremely efficient in purely adaptive terms. Intelligence may have emerged as a form of adaptation to the environment (Piaget 1977), but its efficiency will be mediated by its power of revealing reality to the "adapting organism" in the most adequate form.

The distinction between truth and falsehood refers to the judgment about reality that an intelligent subject is capable of emitting. However, if its notion of truth merely evoked a form of adaption into a specific biological niche (as vast as it may seem), its possibility of effective adaptation to reality would face serious limitations. The onerous shadow of previous forms of adaptation would determine the implementation of new "adjustments" to unexplored environments. The advantage of knowing the "truth" about the world with increasing degrees of insight, clarity, and accuracy resides in the huge number of "degrees of freedom" that it grants: since the individual can know how the environment actually is, it is therefore capable of gaining independence from environmental constraints, and it can 
achieve a more efficient adaptation not only to the environment itself but also to new potential environmental contexts that may eventually appear.

According to current scientific evidence, it seems honest to admit that intelligence has emerged as the result of a series of progressive adaptations of organisms to the environment. One of these adaptations, affecting the most complex of all known organisms, possesses such a high degree of efficiency that it is not limited to fulfilling a specific biological role. Rather, it shapes the organism in such a way that it can apprehend the structure of the environment as such. The grasping of the intelligibility of the world stems from the highly efficient adaption experienced by a very complex species (Homo sapiens sapiens). Nevertheless, through this adaption, the realm of "truth" (defined as consonance or correspondence between the structure adopted by a certain organism in order to satisfy its biological needs and the structure of the environment itself) has been unveiled. The possibility of apprehending reality from a more "universal" perspective, no longer limited to a specific "point of view", such as that of metabolism ${ }^{12}$, but open to "all points of view" (as intelligence enables us to do) bestows upon us a higher degree of autonomy from the environment and it allows for the development of a much more versatile inner world.

In summary, the distinction between truth and falsehood cannot be reduced to evolutionary forces in the epistemological realm. Epistemologically, it has to precede evolutionary forces, which must have acted as necessary but not sufficient conditions for the emergence of our perception of truth and falsehood. Otherwise, no true theory about evolutionary forces could be offered. Because it is utterly implausible to deny the "ontological" influx of evolutionary theories upon the emergence of those psychic faculties which allow us to grasp truth and falsehood, we seem compelled to accept the following thesis:

12 Any adaptation unfolds a certain dimension of reality. The metabolism of amoebae reflects something "universal", but not in the same way as human intelligence, which can commensurate itself to all other forms of adaptation through a magnificent instrument: the power of understanding. 
Evolution has led us ${ }^{13}$ into a situation in which we can apprehend, with increasing degrees of complexity, reality as it is (as manifested by the outstanding progress in the realm of natural sciences and in our knowledge of ourselves); but reality "as it is", its intelligibility, precedes (both temporally and logically) or at least accompanies the display of those evolutionary forces which have paved the path for the possibility that human beings grasp the truth about reality.

\section{Concluding remarks}

Any theory, including Darwinian paradigms, must be susceptible to truth and falsehood. If the distinction between truth and falsehood were subordinated to a certain conceptual frame offered by a particular theory, instead of a scientific theory we would find a meta-scientific explanation (captive to a dangerous spiral of "epistemological totalitarianism"), equivalent to a set of dogmatic, metaphysical claims which block the quest of truth. If a scientific theory submits itself to criteria of scientific falsifiability, it recognizes the priority and irreducibility of the distinction between truth and falsehood. If the theory of evolution is true, it is due to its susceptibility to being true or false. Therefore, it is epistemologically contingent. The only epistemological necessity points to the distinction between the true and the false, that is to say: to the intelligibility of the world, which appears as "true" to a conscious being capable of distinguishing truth from falseness.

The only plausible explanation which makes evolutionary forces compatible with the epistemological autonomy of truth defends that biological

13 Has this process been gradual or abrupt? The distinction between truth and falseness cannot be gradual (our perception of reality can certainly be gradual, but our distinction between truth and falsehood cannot, for there is a "critical point" at which the true differs from the false): we must have reached a level in the development of our evolutionarily shaped capacities for the perception of reality in which a dichotomy between truth and falseness emerged. Saltationism seems more plausible than mere gradualism. Just as the origin of life appears to be the result of a happy, hardly predictable accident, the emergence of higher psychic capacities (language, consciousness...) could have faced some critical point prompted by a sudden genetic change. 
adaption has led us (gradually or through sudden leaps? It is hard to elucidate) into a situation in which we are capable of recognizing the distinction between truth and falsehood. However, truth and falsehood do not depend upon evolutionary forces but upon the intelligibility of the world, which is preexistent or at least "concomitant" to the action of evolutionary forces: it consists of the world itself inasmuch as it becomes manifest to a being that possesses higher intellectual powers. An efficient adaption has propitiated the "unveiling" of truth and falsehood through the endowment of a sufficiently highly developed brain; we do not know if through a happy "cosmic coincidence" (Bedke 2009) or an inexorable, gradual process that guides life into achieving higher degrees of complexity.

The reconciliation of the primacy (in Darwin's theory) of biological efficiency as the natural driving force of evolutionary processes with the necessity of admitting scientific truth (understood not as efficiency but as congruency between a mental formulation and the real phenomenon addressed by our statement) demands accepting that evolutionary development itself has led, through mechanisms still in need of a more refined scientific elucidation, to the emergence of a series of mental capacities structured in such a way that we can recognize the patterns ruling nature. In this model, efficiency is subsidiary: the key point lies in realizing that the human mind has acquired a structure and a set of functional powers of a dimension so that, beyond its "idiosyncrasy", they can commensurate themselves to the structure of nature in a marvelously adequate form. This imperfect but indisputable isomorphism or parallelism between mind and nature allows us to state that the notion of truth (from the side of mind) is as valid as the idea of efficiency (from the side of biological nature). Denying this claim would imply to resuscitate some form of idealism, and I cannot believe that those who support a naturalistic explanation would ever claim to adhere to this option. 


\section{References}

Allen, R.J., and B.R. Leiter. 2001. "Naturalized Epistemology and the Law of Evidence.” Virginia Law Review 87: 1491.

Ball, P. 2013. "DNA: Celebrate the unknowns.” Nature 496 (7446): 419-420.

Bedke, M. 2009. “Intuitive non-naturalism meets cosmic coincidence.” Pacific Philosophical Quarterly 90/2: 188-209.

Bradie, M., and W. Harms. 2011. "Evolutionary epistemology.” In The Stanford Encyclopedia of Philosophy (Winter 2012 Edition), edited by Edward N. Zalta. URL = <http://plato.stanford.edu/archives/win2012/entries/epistemology-evolutionary/>

Braudel, F. 1958. "Histoire et sciences sociales: La longue durée.” Annales. Histoire, Sciences Sociales 13/4 EHESS.

Cantor, G. 1915. Contributions to the Founding of the Theory of Transfinite Numbers. New York: Dover.

Copp, D. 2008. “Darwinian skepticism about moral realism.” Philosophical Issues 18/1: 186-206.

Darwin, Ch. 2009. The Origin of Species by means of Natural Selection: or, the Preservation of Favoured Races in the Struggle for Life, edited by William F. Bynum. London: Penguin Classics.

Dworkin, R. 1996. “Objectivity and truth: You'd better believe it.” Philosophy \& Public Affairs 25/2: 87-139.

Enoch, D. 2011. Taking Morality Seriously: A Defense of Robust Realism. Oxford: Oxford University Press.

Feyerabend, P. 1975. Against Method: Outline of an Anarchistic Theory of Knowledge. Atlantic Highlands NJ: Humanities Press.

Gödel, K. 1931. "Über formal unentscheidbareSätze der Principia Mathematica und verwandterSysteme I”. Monatsheftefürmathematik und physik 38/1: 173-198.

Goethe, J.W von. 1783. “Die Natur.” GoethesWerke. Hamburger Ausgabe in 14 (1994): $45 \mathrm{ff}$.

Gould, S.J., and R.C. Lewontin. 1979. "The spandrels of San Marco and the Panglossian paradigm: a critique of the adaptationist programme." Proceedings of the Royal Society of London. Series B. Biological Sciences 205 (1161): 581-598.

Kahane, G. 2011. “Evolutionary debunking arguments.” Noûs 45/1: 103-125.

Ketelaar, T., and B.J. Ellis. 2000. "Are evolutionary explanations unfalsifiable? Evolutionary psychology and the Lakatosian philosophy of science." Psychological Inquiry 11/1: 1-21. 
Kirschner, M.W., and J.C. Gerhart. 2005. The Plausibility of Life: Resolving Darwin's Dilemma. New Haven: Yale University Press.

Lucas, J.R. 1961. “Minds, machines and Gödel.” Philosophy 36/137: 112-127.

Nagel, T. 1989. The View from Nowhere. Oxford: Oxford University Press.

-. 2012. Mind and Cosmos: Why the Materialist Neo-Darwinian Conception of Nature is almost Certainly False. Oxford: Oxford University Press.

Piaget, J. 1977. La Naissance de l'Intelligence chez l'Enfant. Paris: Delachaux et Niestlé. Popper, K.R. 1935. Logik der Forschung: zur Erkenntnistheorie der modernen Naturwissenschaft. Berlin: Springer.

Shafer-Landau, R. 2003. Moral Realism: A Defence. Oxford: Clarendon Press.

Sols, F. 2014. “Uncertainty, Incompleteness, Chance, and Design.” In Intelligible Design. A Realistic Approach to the Philosophy and History of Science, edited by M.M. Carreira and J.A. Gonzalo, 98-118. New Jersey: World Scientific.

Sperber, D., ed. 2000. Metarepresentations: A Multidisciplinary Perspective. Oxford: Oxford University Press.

Stamos, D. N. 1996. “Popper, falsifiability, and evolutionary biology.” Biology and Philosophy 11/2: 161-191.

Street, S. 2006. “A Darwinian dilemma for realist theories of value.” Philosophical Studies 127/1: 109-166.

Von Uexküll, J. 1909. Umwelt und Innenwelt der Tiere. Berlin: Springer. 